\title{
Long Exposure to CdS-Dextrin Nanoparticles Induces an Immunomodulatory and Anti-Inflammatory Effect in Rats
}

\author{
Gómez-Cansino $\mathrm{R}^{1}$, Reyes-Esparza JA ${ }^{1}$, Rodríguez-Fragoso $\mathrm{P}^{2}$, González de la Cruz $\mathrm{G}^{2}$ and Rodríguez- \\ Fragoso $\mathrm{L}^{* 1}$
}

${ }^{1}$ Facultad de Farmacia, Universidad Autónoma del Estado de Morelos, Cuernavaca, Morelos, Mexico

${ }^{2}$ Departamento de Física CINVESTAV- I.P.N, Ciudad de México, Mexico

${ }^{*}$ Corresponding author: Rodríguez-Fragoso L, Facultad de Farmacia, Universidad Autónoma del Estado de Morelos, Col. Chamilpa, Cuernavaca, Morelos, Mexico, Tel: 7773297000 2321, E-mail: mrodriguezf@uaem. $\mathrm{mx}$

Citation: Gómez-Cansino R, Reyes-Esparza J, Rodríguez-Fragoso P, González de la Cruz G and RodríguezFragoso L (2017) Long Exposure to Cds-Dextrin Nanoparticles Induces an Immunomodulatory and AntiInflammatory Effect in Rats. J Mater Sci Nanotechnol 5(1): 105. doi: 10.15744/2348-9812.5.105

Received Date: May 15, 2017 Accepted Date: June 07, 2017 Published Date: June 13, 2017

\begin{abstract}
Male Wistar rats were treated daily and intraperitoneally with CdS-Dextrin nanoparticles $(100 \mu \mathrm{g} / \mathrm{kg})$ during 30, 60, and 90 days. The effect of subacute and chronic administration of CdS-Dextrin nanoparticles on CD4/CD8 subpopulations of thymocytes, spleenderived T cells, and peripheral blood CD4/CD8/CD3 T cells were analyzed by flow cytometer using a Rat T Lymphocyte Cocktail. An indirect ELISA kit analyzed cytokine Th1/Th2 levels. A DNA content and cell cycle analysis was carried out in bone marrow cells by flow cytometry. We also analyzed the presence of CdS-Dextrin nanoparticles in the thymus and spleen.

CdS-Dx/QDs led to a significant reduction in $\mathrm{CD}^{+}(17 \%)$ and $\mathrm{CD}^{+}(13.7 \%)$ thymocytes at 90 and 60 days, respectively. An increase in $\mathrm{CD}^{+}$spleen-derived cells was also observed. Peripheral blood cells showed an increase in $\mathrm{CD} 4^{+}(28 \%), \mathrm{CD} 8^{+}(25 \%)$ and $\mathrm{CD} 3^{+}$ $(27 \%)$ at 60 days of treatment. However, at 90 days, there was an important decrease in CD4 $4^{+}(40 \%), \mathrm{CD}^{+}(60 \%)$ and $\mathrm{CD} 8^{+}(45 \%)$. No changes were observed in G0/G1 and S phases of cell cycle in bone marrow cells, but there were slight changes in G2+M. The morphological analysis did not show changes in the thymus and spleen, even though the presence of fluorescence attested to the uptake of nanoparticles. Fluorescence imaging revealed a greater uptake of nanoparticles in the spleen, located in the red pulp.

Our results indicate that CdS-Dextrin nanoparticles interact with secondary lymphoid organs, modifying cell populations and the levels of soluble mediators in the immune system. They can induce an immunomodulatory and anti-inflammatory effect that is timedependent.

Keywords: Nanoparticles; Thymocytes; Lymphocytes; Cell cycle; Citokines

List of Abbreviations: BGP: $\beta$-glycerol phosphate; C: Cortex; CdS-Dx/QDs: Cadmium sulfide capped whit dextrin quantum dots; CdSe/ZnS QDs: Zinc sulfide-coated cadmium selenide quantum dots; DTT: Dithiothreitol; EDTA: Ethylenediaminetetraacetic acid; EGTA: Glycol ether diamine tetraacetic acid; H\&E: Hematoxylin and eosin; HEPES: 4-(2-hydroxyethyl)-1-piperazineethanesulfonic acid; IL-4: Interleukin 4; IL-10: Interleukin 10; i.p: Intraperitoneal administration route; IFN- $\gamma$ : Gamma Interferon; $\mathrm{K}_{2} \mathrm{CO}_{3}: \mathrm{Potassium}$ carbonate; M: Medulla; Mz: Marginal zone; NaF: Sodium fluoride; $\mathrm{NH}_{4} \mathrm{Cl}$ : Ammonium chloride; NK: Natural Killer; NP: Nanoparticles; PBS: Phosphate Buffered Saline; PFSM: Phenylmethylsulfonyl fluoride; ROS: Reactive oxygen species; Rp: Red pulp; $\mathrm{TiO}_{2}$ : Titanium oxide; TNF- $\alpha$ : Tumor Necrosis Factor- $\alpha$; Wp: White pulp
\end{abstract}

\section{Introduction}

Advances in nanotechnology have increased the number of toxicological studies meant to understand and predict the effect nanomaterials have in human beings [1-3]. Several studies have shown that, when they come into contact with human cell lines or animal tissue, nanomaterials induce different responses that are basically dependent on their physicochemical and optical properties, as well as the biological environment. These studies serve to lay the foundations for the secure use of nanomaterials [4-8].

Current nanotechnological research is focused on developing biocompatible nanomaterials that can be incorporated into cellular microenvironments and perform their specific function with minimal damage or without an adverse response in the biological system. However, the efficacy of a nanomaterial can be affected depending on whether a reaction in the organism triggers an acute innate immune inflammatory reaction, chronic inflammation, etc. [9-12]. 
Several studies show nanomaterials can stimulate or suppress the immunological response, and there is evidence that their immunogenicity depends largely on their physicochemical characteristics; e.g., size, shape, charge, composition, the chemical groups on the surface, coating, and the state of the biological environment [13-18]. It is currently a difficult task to predict how the immunological system will respond to a determined nanomaterial and if the response will be the same in a healthy or diseased condition considering possible theranostic uses. According to recent reports, there is no doubt that once a nanomaterial enters the body it can be identified as a foreign element, triggering an immune response. The magnitude of this response, however, will depend on multiple factors [7,19].

Our group recently synthesized dextrin-coated cadmium sulfide nanoparticles (CdS-Dextrin) and observed they induce selective cytotoxicity in vitro but are biocompatible in experimental animals. We also found they were widely distributed across different tissues and did not induce damage, either after a single dose $(18 \mathrm{~h})$ or multiples dosages (1 week) [20]. Even if we did not observe tissue damage or clinical signs of toxicity after acute exposure to CdS-Dextrin, we still do not know if CdS-Dextrin nanoparticles can trigger an immune response after a long exposure period. Therefore, the aim of this study was to analyze the effect of subacute and chronic administration of CdS-Dextrin nanoparticles on different components of the immune system such as CD4/ CD8 subpopulations of thymocytes, spleen-derived T cells, peripheral blood CD4/CD8/CD3 T cells, and Th1/Th2 cytokines. Additionally, we analyzed tissues using fluorescence imaging and quantitation of CdS-Dextrin nanoparticles in the thymus and spleen of rats following subacute and chronic administration.

\section{Materials and Methods}

\section{Synthesis}

Cadmium sulfide nanoparticles were prepared in aqueous solution. $\mathrm{CdCl}_{2}(5 \mathrm{~mL}, 0.02 \mathrm{M}), \mathrm{KOH}(10 \mathrm{~mL}, 0.5 \mathrm{M}), \mathrm{NH}_{4} \mathrm{NO}_{3}(5 \mathrm{~mL}$, $0.5 \mathrm{M})$, and $\mathrm{CS}\left(\mathrm{NH}_{2}\right)_{2}(5 \mathrm{~mL}, 0.2 \mathrm{M})$ were added and the mixture was stirred and heated at $80^{\circ} \mathrm{C}$. Similar conditions were applied to maltodextrin with $3 \%$ concentration. These solutions were slowly added into the flask and adjusted to $\mathrm{pH} 11 \mathrm{using}$ a dilute solution of sodium hydroxide. The solution immediately turned a light yellow color, indicating the initial formation of a CdS nanoparticle. The temperature of the mixture was kept at $75^{\circ} \mathrm{C}$ and maintained at this temperature for $60 \mathrm{~min}$. The nanoparticles were separated from the chemical reaction by centrifugation at $6000 \mathrm{rpm}$ during $60 \mathrm{~min}$, and deposited in solid form from the solution; finally, they were washed several times with deionized water and dried at $40{ }^{\circ} \mathrm{C}$ for $24 \mathrm{~h}$. In the dextrin solution, the hydroxyl groups acted as stabilizer agents for the synthesized CdS nanoparticles.

\section{Animals and Treatments}

Bright green emitting CdS-Dextrin nanoparticles (3nm size) were synthesized as previously described [20]. Male Wistar rats (8-10 weeks old) were purchased from Envigo Laboratories Inc. (Mexico) and were allowed to adapt to the animal room conditions for 1 week prior to the beginning of the study. The environmental conditions were controlled (temperature, relative humidity, and a 12 $\mathrm{h}$ light/dark cycle) and the animals were allowed food (Standard Purina Chow Diet, Mexico) and water ad libitum. All the animals used in this study were cared for in accordance with the principles outlined in the "Guide for the Care and Use of Laboratory Animals" [21].

Healthy Wistar male rats were randomly selected and divided into four groups (4 rats for each group): three groups were treated with CdS-Dextrin nanoparticles at $100 \mu \mathrm{g} / \mathrm{kg}$ i.p. daily during 30, 60 or 90 days respectively, and the fourth group (control) received $200 \mu \mathrm{L}$ of PBS solution daily. Toxicity indices consisted of daily clinical observations regarding body weight, food consumption, clinical pathology, organ weights, and histopathology. At the end of treatment, animals were fasted overnight and blood samples were obtained from their heart following anesthesia with ether. The spleen and thymus were collected and divided: one part was preserved in 10\% neutral-buffered formalin fixative and processed for routine histological examination, while the other was used for the quantitative analysis of CdS-Dextrin nanoparticles and imaging. A histopathological analysis was performed using standard laboratory procedures. The spleen and thymus tissues were fixed in a 10\% formalin solution for one week. Tissues were embedded in paraffin blocks, and sectioned into $5 \mu \mathrm{m}$ slices, mounted on glass slides and stained with hematoxylin-eosin (H\&E).

\section{Analysis of Cell Populations from the Thymus, Spleen and Peripheral Blood}

Single-cell lymphocyte populations were prepared from the thymus, spleen and peripheral blood of rats. Tissues were dissociated on a steel mesh with RPMI 1640 medium. The collected tissue suspension was centrifuged at $2000 \mathrm{rpm}$ for 5 min. The supernatant was discarded and cells were resuspended in the RPMI 1640 medium. A lysis buffer $\left(0.15 \mathrm{M} \mathrm{NH}_{4} \mathrm{Cl}, 0.1 \mathrm{mM}\right.$ EDTA and 9.9 mM $\mathrm{K}_{2} \mathrm{CO}_{3}$ ) was added in order to induce the lysis of red blood cells. Then, the cell suspension was filtered and washed to remove cell debris. Finally, cells were resuspended in RPMI 1640 medium. Cell viability was assessed using the trypan blue exclusion test and routinely found to contain $<5 \%$ dead cells. In order to identify the cell populations (CD4/CD 8 subpopulations of thymocytes, spleen-derived T cells, and peripheral blood CD4/CD8/CD3 T cells) the cell density was adjusted to $5 \times 10^{5}$ cells/mL and analyzed by flow cytometry using $10 \mu \mathrm{L}$ of Rat T Lymphocyte Cocktail (558493, BD Pharmingen ${ }^{\mathrm{Tm}}$ ) in a 1:5 dilution. 


\section{Analysis of DNA Content and Cell Cycle Analysis in Bone Marrow Cells}

Cell cycle analysis and DNA content measurement were performed according with the following procedure. $1 \mathrm{x} 10^{5} \mathrm{cells}$ per $\mathrm{mL}$ were fixed in $75 \%$ ethanol for $24 \mathrm{~h}$, then washed in PBS and resuspended in $0.1 \%$ Nonidet P40 (Biochemica Fluka) and DNasefree RNase $\left(10 \mathrm{mgmL}^{-1}\right)$ for $20 \mathrm{~min}$ at room temperature [22]. Propidium Iodide was then added (final concentration $5 \mathrm{mg} / \mathrm{mL}$ ) and incubated for $12 \mathrm{~h}$ at $4{ }^{\circ} \mathrm{C}$ in the dark. Samples were analyzed using a FACScalibur flow cytometer (Becton Dickinson). For each sample, 10,000 cells were analyzed using four replicates. The results were analyzed using the CELLQuest DNA experiment software.

\section{Analysis of The Effect of CdS-Dextrin Nanoparticles on Th1/Th2 Cytokine Serum Levels}

Serum samples were obtained from peripheral blood by centrifugation at $3000 \mathrm{rpm}$ for $15 \mathrm{~min}$ at $4{ }^{\circ} \mathrm{C}$ and immediately frozen at $-80^{\circ} \mathrm{C}$ for further detection. IL-6, TNF- $\alpha$, IFN- $\gamma$, IL-4, and IL-10 levels were analyzed by an indirect ELISA in serum [23]. Wells were coated with $100 \mu \mathrm{L}$ of serum sample and incubated overnight at $4{ }^{\circ} \mathrm{C}$. Wells were aspirated, washed and blocked with $150 \mu \mathrm{L}$ of casein $5 \%$ for $60 \mathrm{~min}$ at room temperature. After washing, $100 \mu \mathrm{L}$ of primary antibody (dilution 1:1000) were added to each well and incubated for $60 \mathrm{~min}$ (IL-4, IL- 6 and IFN- $\gamma$ antibodies) or $120 \mathrm{~min}$ (TNF- $\alpha$ and IL-10 antibodies) at room temperature, followed by washing and an added $100 \mu \mathrm{L}$ of secondary antibody (dilution 1:1500) incubated for 60 min at room temperature. After washing, $100 \mu \mathrm{L}$ of substrate were added to each well for detection and incubated for 30 or 60 min at room temperature. Samples were quantified in a Multimode Plate Reader Victor X3, Perkin Elmer (USA) at $405 \mathrm{~nm}$.

\section{Analysis of CdS-Dextrin Nanoparticles Distribution in the Thymus and Spleen}

To obtain precise images of QD distribution in the thymus and spleen, unstained slices of tissue samples were analyzed under a fluorescence microscope. In order to reduce autofluorescence, the tissue samples were rinsed many times with $1 \mathrm{mg} / \mathrm{mL}$ solution of sodium borohydride as a blocking agent. We also performed a quantitative analysis of CdS-Dextrin nanoparticles. For this, 50 mg of tissue were homogenized with $1 \mathrm{~mL}$ of lysis buffer (20mM HEPES, $2 \mathrm{mM}$ EGTA, $50 \mathrm{mM} \beta$-glycerol phosphate, $5 \mathrm{mM}$ sodium fluoride, $50 \mu \mathrm{M}$ dithiothreitol (DTT), $100 \mathrm{mM}$ phenylmethylsulfonyl fluoride (PMSF)). The intensity of fluorescence emitted by tissues was measured using a spectrophotometer (Perkin Elmer) with the excitation wavelength of $485 \mathrm{~nm}$.

\section{Statistical Analysis}

Statistical analysis was done using GraphPad Prism version 7.00 for Windows (La Jolla California USA, www.graphpad.com). One-way ANOVA was performed, followed by Tukey multiple comparisons test. Differences were considered significant if the P value was less than 0.05 .

\section{Results}

\section{Effect of CdS-Dextrin Nanoparticles on CD4/CD8 Subsets of Thymocytes, Spleen-Derived T Cells, and Peripheral Blood CD4/CD8 T Cells}

Figure 1 shows the observed changes in the cell populations of the thymus, spleen and peripheral blood induced by multiple
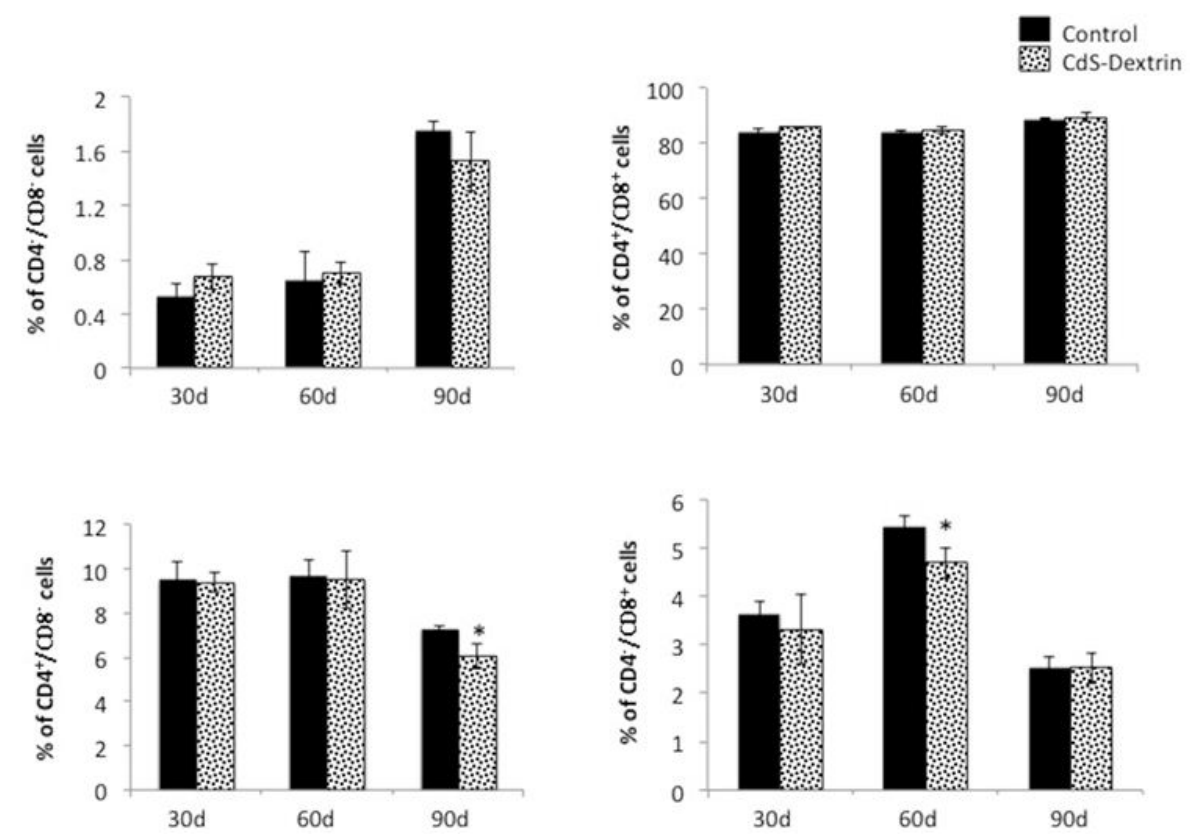

Figure 1: Effect of CdS-Dextrin nanoparticles on CD4/CD8 subsets of thymocytes. Animals were treated with CdS-Dextrin nanoparticles $(100 \mu \mathrm{g} / \mathrm{kg})$ administered via i.p. during 30, 60 and 90 days. Results are represented as mean \pm d.s. $n=4$, ${ }^{*}$ represents significantly different data from the control group $(p<0.05)$ 
subacute and chronic dosages of CdS-Dextrin nanoparticles $(100 \mu \mathrm{g} / \mathrm{kg})$. Non-significant changes were observed in the double negative (lineage-negative) and double positive thymocytes of any of the groups at any time during the study. A significant decrease in the population of single positive $\mathrm{CD} 4\left(\mathrm{CD}^{+} \mathrm{CD}^{-}\right)(10.9 \%)$ thymocytes was observed in rats treated with CdS-Dextrin nanoparticles during 90 days $(\mathrm{p}<0.05)$. We also observed a significant decrease of single positive CD8 $\left(\mathrm{CD} 4 \mathrm{CD}^{+}\right)$thymocytes in rats treated during 60 days $(17 \%)(\mathrm{p}<0.05)$. The spleen showed a significant increase of CD4 $4^{+} \mathrm{T}$ cells $(13.7 \%)$ in the group treated with CdS-Dextrin nanoparticles for 60 days $(\mathrm{p}<0.05)$, but no changes were observed at 30 and 90 days. No significant changes were detected in the CD8 ${ }^{+} \mathrm{T}$ cells of animals treated with CdS-Dextrin nanoparticles at any time during the study (Figure 2). CD4, CD8 and CD3 T cells were studied in peripheral blood at 60 and 90 days. We found that all subpopulations of cells had significantly increased after 60 days of treatment with CdS-Dextrin nanoparticles (CD4 (28\%), CD8 (25\%) and CD3 (27\%)) (p<0.05). However, all cell subpopulations had a significant decrease at 90 days of exposure (CD4 (40\%), CD8 (60\%) and CD3 (45\%) (p<0.05) (Figure 3).
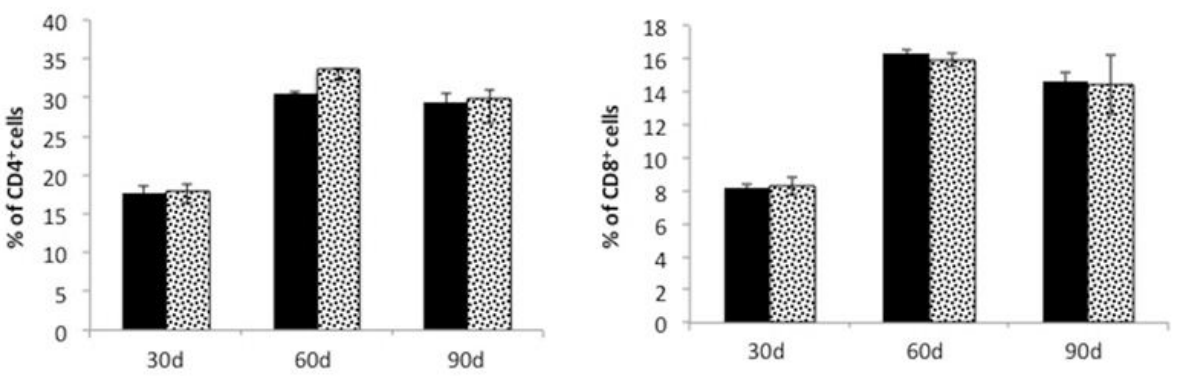

Figure 2: Effect of CdS-Dextrin nanoparticles on spleen-derived T cells. Animals were treated with CdSDextrin nanoparticles $(100 \mu \mathrm{g} / \mathrm{kg})$ administered via i.p. during 30,60 and 90 days. Results are represented as mean \pm d.s. $\mathrm{n}=4,{ }^{*}$ represents significantly different data from the control group $(p<0.05)$
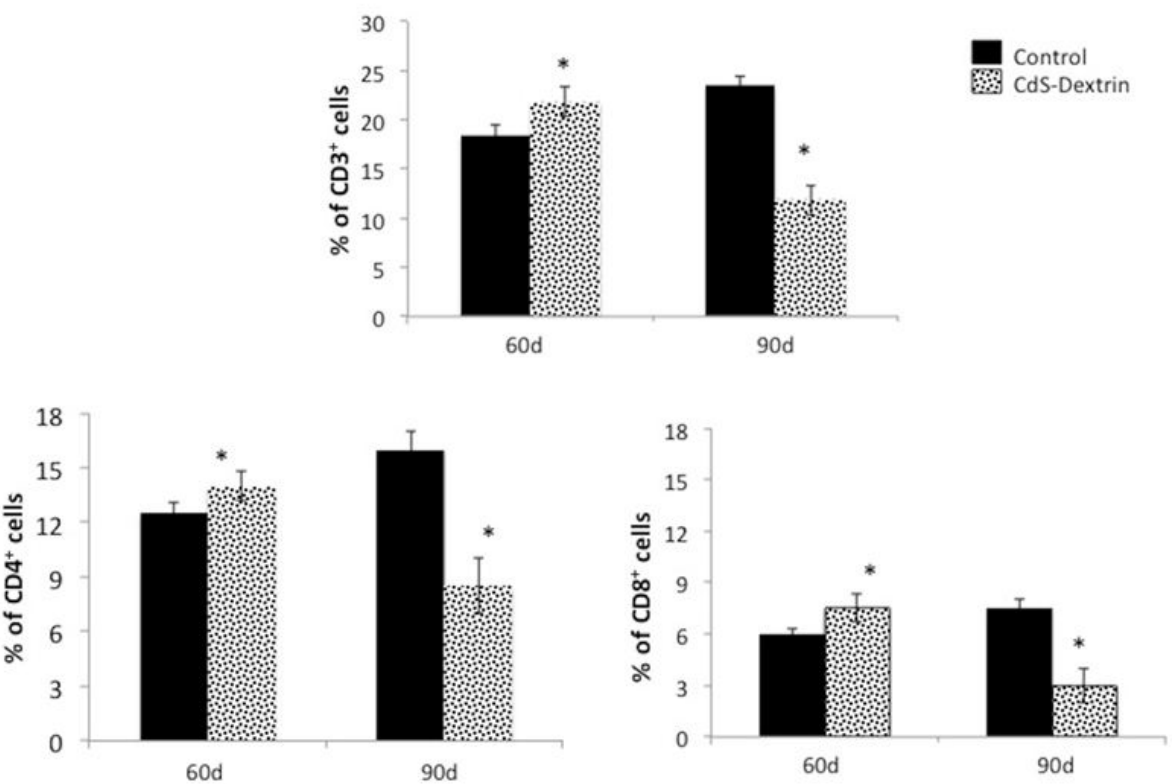

Figure 3: Effect of CdS-Dextrin nanoparticles on CD4/CD8 peripheral blood CD4/CD8 T cells. Animals were treated with CdS-Dextrin nanoparticles $(100 \mu \mathrm{g} / \mathrm{kg})$ administered via i.p. during 60 and 90 days. Results are represented as mean \pm d.s. $n=4,{ }^{*}$ represents significantly different data from the control group $(p<0.05)$

\section{Effect of CdS-Dextrin Nanoparticles on Th1/Th2 Cytokine Levels in Serum}

Figure 4 shows the observed changes in rat serum cytokine levels after multiple dosages of CdS-Dextrin nanoparticles. There was a significant increase on Th1 cytokine levels; TNF- $\alpha$ and IL- 6 were detected after 30 days of treatment with CdS-Dextrin nanoparticles and we found a significant increase $(89.15 \%$ and $34.29 \%$ respectively $(\mathrm{p}<0.05)$, and non-significant changes were observed after 60 and 90 days of treatment in both cytokines. Interestingly, IFN- $\gamma$ levels only increased at 30 and 60 days (27.05\% and $34.59 \%$, respectively) ( $\mathrm{p}<0.05)$. On the other hand, Th2-cytokines increased considerably. IL-4 increased by $98.05 \%, 63.10 \%$ and $60.77 \%$ at 30,60 and 90 days respectively $(\mathrm{p}<0.05)$ when compared with the control group. IL-10 levels increased by $39.19 \%$, $18.65 \%$ and $30.60 \%$ at 30,60 and 90 days respectively $(\mathrm{p}<0.05)$ when compared with the control group. 

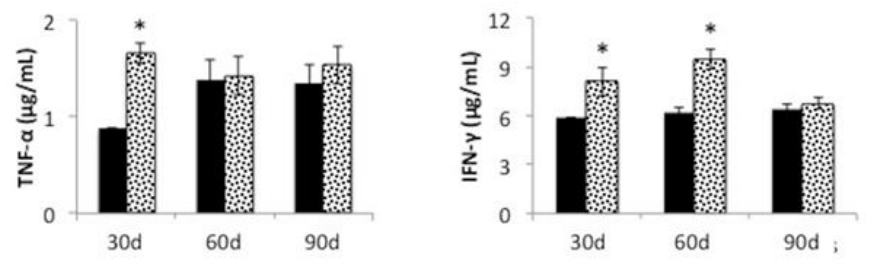

Control

CdS-Dextrin
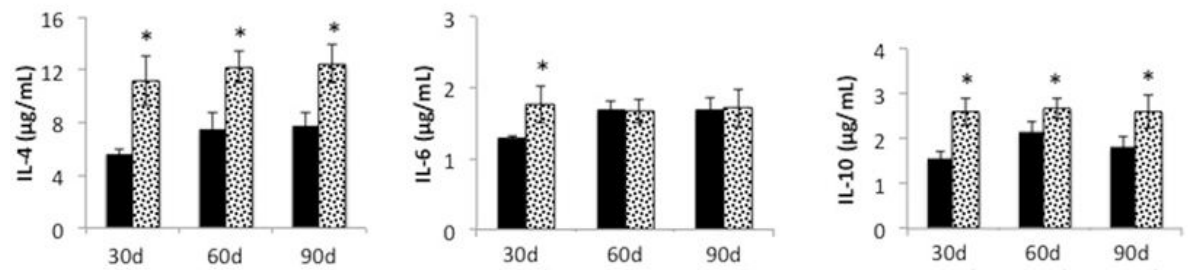

Figure 4: Effect of CdS-Dextrin nanoparticles on Th1/Th2 cytokine levels in serum. Animals were treated with CdS-Dextrin nanoparticles $(100 \mu \mathrm{g} / \mathrm{kg})$ administered via i.p. during 30, 60 and 90 days. Results are represented as mean \pm d.s. $n=4,{ }^{*}$ represents significantly different data from the control group $(p<0.05)$

\section{Effect of CdS-Dextrin Nanoparticles on the Cell Cycle Analysis of Bone Marrow Cells}

Figure 5 shows cell percentage in different phases of the cell cycle across the population of bone marrow cells collected from rats treated with CdS-Dextrin nanoparticles at 30, 60 and 90 days. Our results show no differences in the G0/G1 and S phases of the cell cycle at 30 and 60 days of treatment. However, there was a significant decrease $(10 \%)$ in the $\mathrm{G} 2+\mathrm{M}$ phase at 30 days (p<0.05), and a significant increase at 90 days $(14 \%)(\mathrm{p}<0.05)$. No changes in DNA content were observed.

G0/G1 Phase

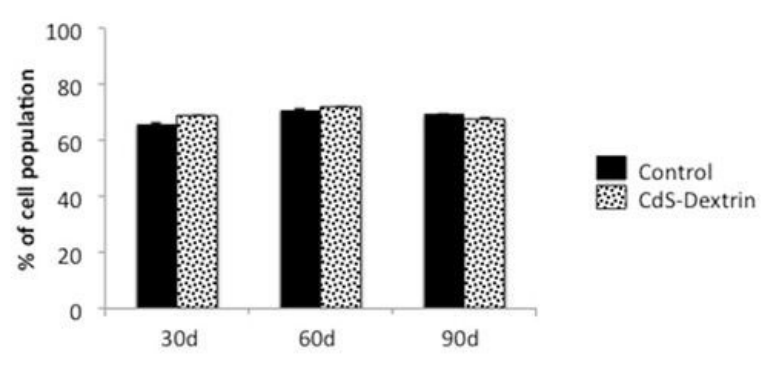

S Phase

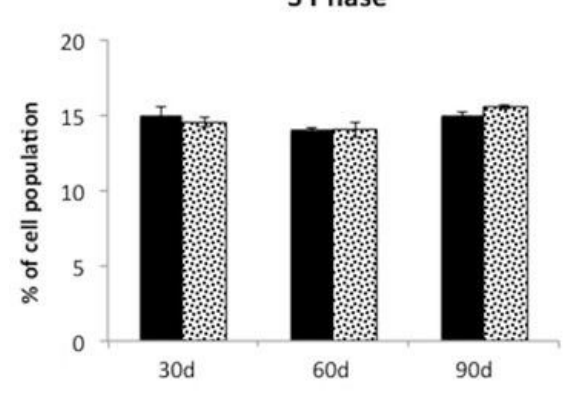

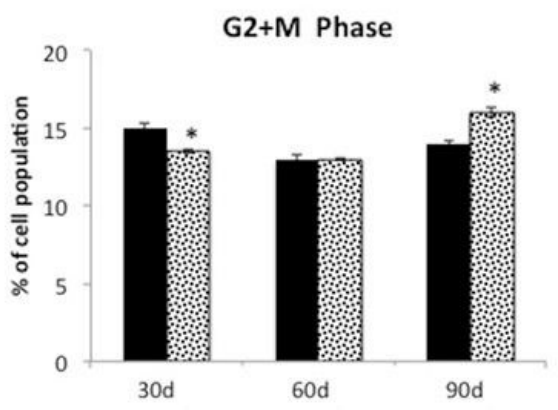

Figure 5: Effect of CdS-Dextrin nanoparticles on cell cycle analysis of bone marrow cells. Animals were treated with CdS-Dextrin nanoparticles $(100 \mu \mathrm{g} / \mathrm{kg})$ administered via i.p. during 30, 60 and 90 days. Results are represented as mean \pm d.s. $n=4,{ }^{*}$ represents significantly different data from the control group $(p<0.05)$

\section{Fluorescent Microscopic Visualization and Quantitative Analysis of the CdS-Dextrin Nanoparticles in the Thymus and Spleen}

To identify the localization of CdS-Dextrin nanoparticles, we analyzed unstained slices from the spleen and thymus under a fluorescence microscope, because CdS-Dextrin nanoparticles emit light at $535 \mathrm{~nm}$ (green fluorescence). Figures 6 and 7 show representative fluorescence images of the tissue distribution and the amounts of CdS-Dextrin nanoparticles in the thymus and spleen at 30, 60 and 90 days of treatment. In order to obtain precise data regarding the uptake of CdS-Dextrin nanoparticles in the thymus and spleen, we quantified the amount of CdS-Dextrin nanoparticles present in those organs using spectrophotometry. The intensity of fluorescence in thymus tissue was minimal; the highest intensity of fluorescence was observed at 60 days. Interestingly, 
there was a significant increase in fluorescence (9-fold) at 60 days when compared with the amount observed at 30 days. The scarce fluorescence was scattered across the thymus tissue; importantly, the degree of fluorescence was observed at 90 days was insignificant (Figure 6). In contrast, the spleen showed a time-dependent increase in the amount of fluorescence induced by CdSDextrin nanoparticles. Fluorescence intensity increased depending on the time span: 99635, 123450, and 177550 AU/mg of tissue. at 30,60 and 90 days respectively $(\mathrm{p}<0.05)$ (Figure 7). The amount of fluorescence in this tissue was much higher than in the thymus: at 60 days it was almost 300-fold higher in the spleen than in the thymus. The presence of intense fluorescence was more evident in red pulp and the transitional zone in the spleen.

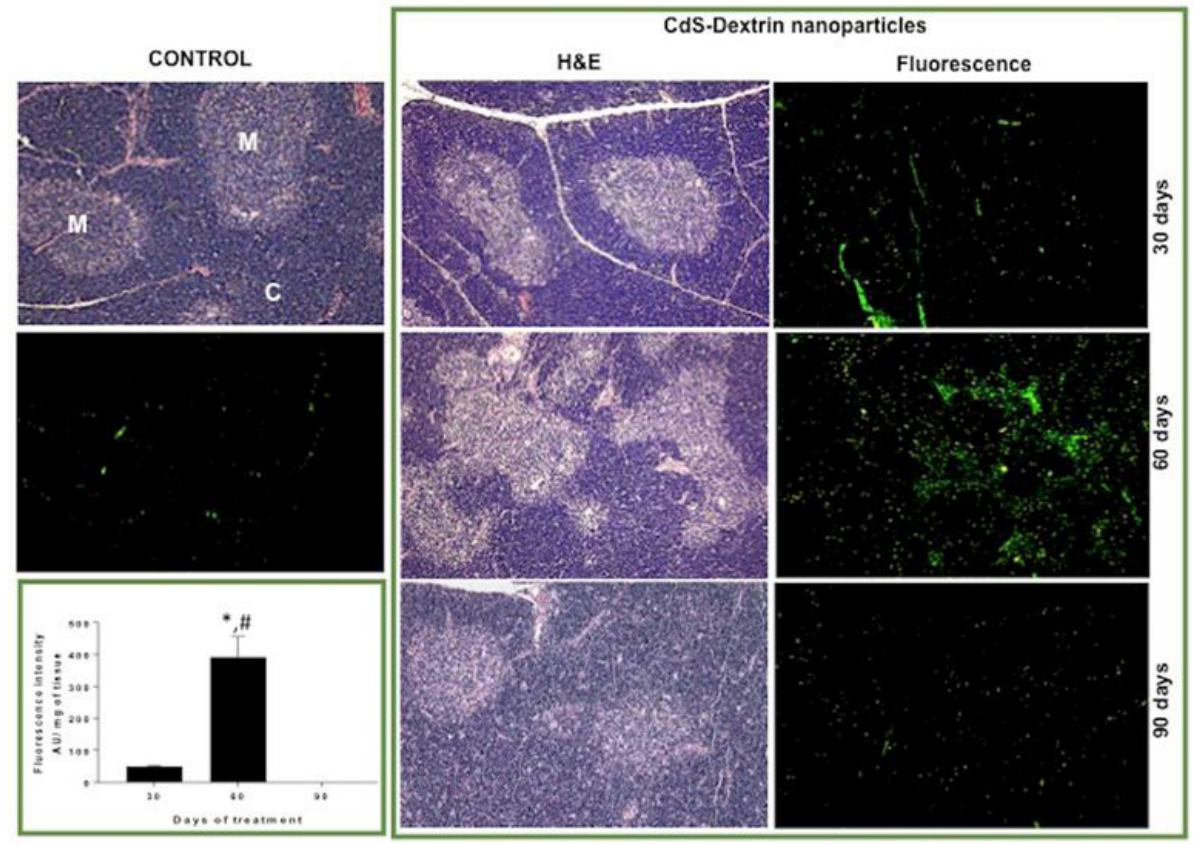

Figure 6: Microscopic visualization and quantitative analysis of CdS-Dextrin nanoparticles in the thymus of rats. Representative fluorescent microscopic images showing the distribution and localization of CdSDextrin nanoparticles in thymus sections from rats treated with $100 \mu \mathrm{g} / \mathrm{kg}$ of CdS-Dextrin nanoparticles administered via i.p. during 30, 60 and 90 days. H\&E stain shows the morphological appearance of the thymus after exposure to nanoparticles (magnification 10X). M (medulla), C (cortex).

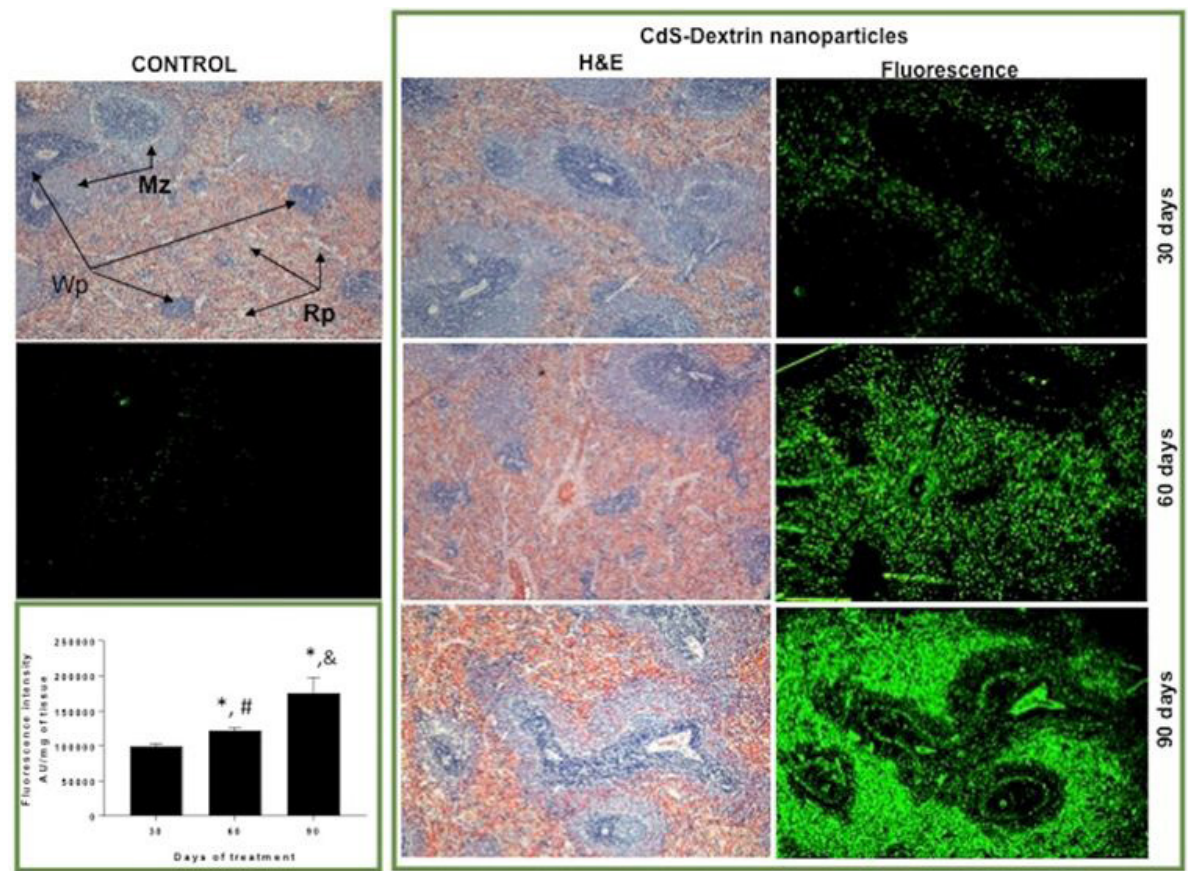

Figure 7: Microscopic visualization and quantitative analysis of CdS-Dextrin nanoparticles in the spleen of rats. Representative fluorescence microscopic images showing the distribution and localization of CdSDextrin nanoparticles in spleen sections from rats treated with $100 \mu \mathrm{g} / \mathrm{kg}$ of CdS-Dextrin nanoparticles administered via i.p. during 30,60 and 90 days; the intensity of fluorescence is evident in the red pulp. H\&E staining shows the morphological appearance of the spleen after exposure to nanoparticles (magnification of 10X). Wp (white pulp), $\mathrm{Rp}$ (red pulp), $\mathrm{Mz}$ (marginal zone). 
A histopathological analysis was carried out in both tissues. At 30 days, thymus morphology was similar in cortex and medulla to that in the control group. There was, however, an increase in the extension and shape of the thymus' medulla at 60 days of treatment. Thymus morphology at 90 days was like the tissues from the control group.

On the other hand, we observed an increase of the marginal zone and a decrease of red pulp in the spleen after 30 and 90 days; at 60 days the morphology was like that of the control group.

\section{Discussion}

Our knowledge regarding the immunogenicity and immunomodulation of nanomaterials has advanced in recent years. Understanding nanomaterial/immune system interactions is a necessary step in the creation of safety norms, especially if nanomaterials are to be employed medically.

Previous studies by this group have shown that CdS-Dextrin nanoparticles are biocompatible in vivo, can be easily distributed in tissues and even cross biological barriers without generating toxicity. Although these nanoparticles have proven to be nontoxic over very short periods of time, we do not yet know if they go unnoticed by the immune system because of their small size ( $3 \mathrm{~nm}$ ) or if they are quickly identified and eliminated by circulating/resident immune cells or the reticuloendothelial system, nor whether they can produce an immunomodulatory effect. Therefore, our present study focused on whether CdS-Dextrin nanoparticles could modify cells, soluble mediators, and interact with organs of the immune system after prolonged exposure in a rat model.

The immune system is a complex combination of organs, cells, and molecules involved in removing foreign substances from the body. In some cases, though, this process becomes self-defeating because it can lead to inflammation or autoimmunity. Nanomaterials by themselves are foreign substances and should be able to trigger an immune response; that said, whether they go unnoticed or interact directly is still debatable. The extant literature shows nanomaterials can exert both favorable and harmful effects on the organism as a result of their interaction with the immune system, and this will basically depend on the properties and coating of nanoparticles [24-27].

This study addressed CD4 and CD8 thymocyte subsets in order to know which populations of mature T lymphocytes would be produced in the thymus in response to the presence of CdS-Dextrin nanoparticles, since this organ plays a crucial role in the cellular immune response and the immune response as a whole. We observed that only CD4+ CD8- (helper T cell) cells decreased at 90 days, whereas CD4-CD8+ cells (cytotoxic T cell) increased at 60 days. In the spleen, there was only a slight increase in the populations of CD4+ and CD8+ cells at 60 days.

The blood showed an increase of $\mathrm{T}$ lymphocytes $\left(\mathrm{CD} 4^{+}\right.$and $\left.\mathrm{CD} 8^{+}\right)$at 60 days. According to cytokine data, this seems to be a consequence of restored balance in these populations. The decrease at 90 days in both subpopulations of T lymphocytes could be due to the fact that the thymus is producing more lymphocytes of these subpopulations through a mechanism we have yet to identify.

Variations in B and T cells after exposition to nanoparticles have already been reported. For example, exposure to CdSe/ZnS QDs induced a decrease in the population of T lymphocytes and an increase in the population of B lymphocytes, but had no effect on the population of NK cells in mice exposed for 42 days [28]. On the other hand, Fu et al. observed that titanium dioxide NPs (nano- $\mathrm{TiO}_{2}$ ) induced proliferation of $\mathrm{B}$ and $\mathrm{T}$ lymphocytes after continued exposure for 4 weeks [29]. Mice treated with TiO2 for 7 days showed a reduction of developing B-lymphocytes (CD21-IgM+ cells) and a reduction in the number of CD4 ${ }^{+}$and $\mathrm{CD}^{+}$ lymphocytes [30]. These results indicate that both prolonged and short exposure to nanoparticles can affect the development and production of immune cells. Several authors have pointed out that the immune system's recognition of nanoparticles is influenced by the type of covering, the formation of the protein corona, the charge, and the functional group, among others. Since each nanomaterial has its own characteristics, it might modulate the immune system's response through several pathways [1,24,31,32].

The secretion of some soluble mediators through which the cells of the immune system communicate coordinates the cellular events involved in the immune response. Studies indicate that, after exposure to nanomaterials, the blood levels of these agents can change. Several reports indicate that nanoparticle exposure triggers changes in Th1 and Th2 cytokine profiles and leads to a response [27,33-35]. The present study evaluated the effect of the CdS-Dextrin nanoparticles on the blood level of Th1 cytokines (TNF- $\alpha$ and INF- $\gamma$ ) and found a significant increase in TNF- $\alpha$ after 30 days of exposure; IFN- $\gamma$ levels had a significant increase at 30 and 60 days. On the other hand, the blood levels of Th2 cytokines (IL-4, IL-6 and IL-10) also showed a significant rise; IL-6 only increased at 30 days, whereas the levels of IL- 4 and IL-10 remained high throughout the 90 days of exposure. During the early stages (30 days), the Th1/Th2 balance appeared stable, whereas in later stages there was a tendency toward the Th2 (anti-inflammatory) response. Th1 cytokines are related to cellular response and activate cytolytic lymphocytes and macrophages. In contrast, Th2 cytokines are related to humoral response and inhibit the activation of macrophages; they also promote the development of allergies and autoimmune diseases [36]. We know that, during an inflammatory process, either Th1 or Th2 lymphocytes can oscillate toward a middle pathway in order to attain balance. The predominance of one will produce a prolonged characteristic state of cellular or humoral defense, which at a given point can lead to inflammatory states acceptable to the host, without producing a manifestation of disease [15]. 
Our study also tested whether CdS-Dextrin nanoparticles could cross the blood-thymic barrier (BTB) and whether this might modify the T-cell maturation process, "directing" it or being directed toward a type of cell line: CD4 or CD8. Interestingly, CdSDextrin nanoparticles managed to cross the barrier in very small amounts, and their presence was 300 times lower than in the spleen, for example. Nanoparticle uptake the thymus was minimal and did not lead to evident changes in the maturation process of thymocytes, since no changes were observed in the subpopulations of double negative or double positive thymocytes in the early stages of maturation. That said, there was an increase in $\mathrm{CD}^{+}$subpopulation at 90 days of exposure, which correlates with an increase in this population of cells in the blood. The blood-thymic barrier prevents the presence of both its own and foreign antigens in the interstitial fluid of the organ, which could affect the maturation of pro-lymphocytes and foster antigen tolerance. We do not know how the uptake of nanoparticles affects the thymus and the subsequent immune response. Microscopic observations suggest they do not induce atrophy of the organ tissue. The thymic barrier is known to prevent "disturbances" of the humoral microenvironment of the thymus, so it is mostly the organ itself that regulates the secretion of cytokines within it. To determine if the presence of nanoparticles altered the pattern of cytokine production, we would have had to identify the molecules in the tissue or analyze their expression.

Previous studies by this group have shown the presence of CdS-Dextrin nanoparticles in brain and testicles, suggesting an ability to cross tissue barriers such as the blood brain barrier (BBB) and the blood-testis barrier (BTB). Apparently, the thymic barrier is more selective and strictly controlled, preventing nanomaterial from crossing into it, our results agreed with previously reported data, indicating the reduced presence of nanoparticles in the thymus [37].

We also assessed CdS-Dextrin nanoparticle uptake in the spleen. This organ lacks a cellular barrier to prevent the passage of nanoparticles and showed a substantial uptake that increased over time. After 90 days of continuous exposure, the fluorescence intensity in the spleen tissue was greater, especially in the red pulp (which houses red cells and lymphocytes) and in transition zone, which house (which houses plasma cells). The presence of nanoparticles was 300 times higher in the spleen than in the thymus. The spleen is involved in several important functions that include the elimination of old erythrocytes, blood reservoir, and sites for the "nesting" and activation of B and T lymphocytes to turn them into immunologically competent defense cells. Previous studies by other groups have also reported a high concentration of nanomaterials in the spleen [37-40].

Exposure to CdS-Dextrin nanoparticles for 30 days affected bone marrow cells, resulting in a decrease of the G2+M phase of the cell, but we observed an increase at 90 days. We do not know the lineage of the cell population that showed this increase: further research is needed to establish if it is the lymphoid population, as that would correlate to the increase in CD8 ${ }^{+} \mathrm{T}$ cells in the thymus.

Given our findings regarding the CD4 and CD8 thymocytes subsets, spleen-derived T cells, as well as the observed blood cytokine profiles, we can conclude that CdS-Dextrin nanoparticles induce an immunomodulatory effect after prolonged exposure in a rat model. However, since we did not find a significant uptake of nanoparticles in the thymus or significant changes in thymocyte subsets, and the only significant alterations were in the serum cytokine profile, we think nanoparticles might be interacting primarily with circulating immune cells. Other studies have mentioned the interaction between nanoparticles and circulating immune cells or secondary lymphoid tissues. In present work, DNA quantification and cell cycle analysis in bone marrow indicated non-significant changes [41,42]. Our results agree with previous reports regarding the presence of an initial state of inflammation; afterwards, however, an anti-inflammatory state predominantes.

\section{Conclusions}

Our results indicate that CdS-Dextrin nanoparticles interact with secondary lymphoid organs, modifying cell populations and the levels of soluble mediators in the immune system; they can induce an immunomodulatory and anti-inflammatory effect that is time-dependent. The present results indicate there is still much to learn about the immunomodulatory effects of CdS-Dextrin nanoparticles as well as others types of nanoparticles. Further research is needed to ensure nanomaterials are safely and efficiently used in biomedicine.

\section{Acknowledgment}

Rocío Gómez Cansino wishes to thank the PhD program in Pharmacology at UAEM and CONACyT for the postdoctoral fellowship.

\section{References}

1. Najafi-Hajivar S, Zakeri-Milani P, Mohammadi H, Niazi M, Soleymani-Goloujeh M, et al. (2016) Overview on experimental models of interactions between nanoparticles and the immune system. Biomed Pharmacother 83: 1365-78.

2. Shatkin JA (2012) Nanotechnology: Health and Environmental Risks, Second Edition CRC Press.

3. Suma RN, Mohanan PV (2015) Stem Cells, a New Generation Model for Predictive Nano Toxicological Assessment. Curr Drug Metab 16: 932-9.

4. Albanese A, Tang PS, Chan WC (2012) The effect of nanoparticle size, shape, and surface chemistry on biological systems. Annu Rev Biomed Eng 14: 1-16.

5. Clift MJ, Gehr P, Rothen-Rutishauser B (2011) Nanotoxicology: a perspective and discussion of whether or not in vitro testing is a valid alternative. Arch Toxicol 85: 723-31.

6. Lankveld DP, Van Loveren H, Baken KA, Vandebriel RJ (2010) In vitro testing for direct immunotoxicity: state of the art. Methods Mol Biol 598: 401-23.

7. Petrarca C, Clemente E, Amato V, Pedata P, Sabbioni E, et al. (2015) Engineered metal based nanoparticles and innate immunity. Clin Mol Allergy 13: 13. 
8. Ali A, Suhail M, Mathew S, Shah MA, Harakeh SM, et al. (2016) Nanomaterial Induced Immune Responses and Cytotoxicity. J Nanosci Nanotechnol 16: 40-57. 9. Dobrovolskaia MA, McNeil SE (2007) Immunological properties of engineered nanomaterials. Nat Nanotechnol 2: 469-78.

10. FDA (2002) Guidance for Industry: Immunotoxicology Evaluation of Investigational New Drugs. In: Services USD o HaH, editor. Rockville, Food and Drug Administration.

11. Grabbe S, Landfester K, Schuppan D, Barz M, Zentel R (2016) Nanoparticles and the immune system: challenges and opportunities. Nanomedicine (Lond) 11: 2621-4.

12. Li Y, Italiani P, Casals E, Valkenborg D, Mertens I, et al. (2016) Assessing the Immunosafety of Engineered Nanoparticles with a Novel in vitro Model Based on Human Primary Monocytes. ACS Appl Mater Interfaces.

13. Ghosh M, Sinha S, Jothiramajayam M, Jana A, Nag A, et al. (2016) Cyto-genotoxicity and oxidative stress induced by zinc oxide nanoparticle in human lymphocyte cells in vitro and Swiss albino male mice in vivo. Food Chem Toxicol 97: 286-96.

14. Grosse S, Stenvik J, Nilsen AM (2016) Iron oxide nanoparticles modulate lipopolysaccharide-induced inflammatory responses in primary human monocytes. Int J Nanomedicine 11: 4625-42.

15. Luo YH, Chang LW, Lin P (2015) Metal-Based Nanoparticles and the Immune System: Activation, Inflammation, and Potential Applications. Biomed Res Int 2015: 143720.

16. Ryman-Rasmussen JP, Riviere JE, Monteiro-Riviere NA (2007) Variables influencing interactions of untargeted quantum dot nanoparticles with skin cells and identification of biochemical modulators. Nano Lett 7: 1344-8.

17. Winkler HC, Suter M, Naegeli H (2016) Critical review of the safety assessment of nano-structured silica additives in food. J Nanobiotechnology 14: 44.

18. Zhang Z, Zhang F, Zhang H (2016) In Vitro Response of Immune Cells on Metal Oxide Nanoparticles with Different Solubility. J Nanosci Nanotechnol 16: 5546-52.

19. Zentel R (2016) Nanoparticles and the immune system: challenges and opportunities. Nanomedicine (Lond) 11: 2619-20.

20. Reyes-Esparza J, Martinez-Mena A, Gutierrez-Sancha I, Rodriguez-Fragoso P, de la Cruz GG, et al. (2015) Synthesis, characterization and biocompatibility of cadmium sulfide nanoparticles capped with dextrin for in vivo and in vitro imaging application. J Nanobiotechnology $13: 83$.

21. Albus U (2012) Guide for the Care and Use of Laboratory Animals (8th edn). Laboratory Animals 46: $267-8$.

22. Darzynkiewicz Z, Juan G, Bedner E (2001) Determining cell cycle stages by flow cytometry. Curr Protoc Cell Biol Chapter 8: Unit 8 .

23. Lin AV (2015) Indirect ELISA. In: Hnasko R, editor. ELISA: Methods and Protocols. New York, NY, Springer New York.

24. Moyano DF, Liu Y, Peer D, Rotello VM (2016) Modulation of Immune Response Using Engineered Nanoparticle Surfaces. Small 12: 76-82.

25. Dimitriou NM, Tsekenis G, Balanikas EC, Pavlopoulou A, Mitsiogianni M, et al. (2017) Gold nanoparticles, radiations and the immune system: Current insights into the physical mechanisms and the biological interactions of this new alliance towards cancer therapy. Pharmacol Ther S0163-7258: 30086-4.

26. Dobrovolskaia MA, Shurin M, Shvedova AA (2016) Current understanding of interactions between nanoparticles and the immune system. Toxicol Appl Pharmacol 299: 78-89.

27. Kononenko V, Narat M, Drobne D (2015) Nanoparticle interaction with the immune system. Arh Hig Rada Toksikol 66: 97-108.

28. Wang X, Tian J, Yong KT, Zhu X, Lin MC, et al. (2016) Immunotoxicity assessment of CdSe/ZnS quantum dots in macrophages, lymphocytes and BALB/c mice. J Nanobiotechnology 14: 10.

29. Fu Y, Zhang Y, Chang X, Zhang Y, Ma S, et al. (2014) Systemic immune effects of titanium dioxide nanoparticles after repeated intratracheal instillation in rat. Int J Mol Sci 15: 6961-73.

30. Moon EY, Yi GH, Kang JS, Lim JS, Kim HM, et al. (2011) An increase in mouse tumor growth by an in vivo immunomodulating effect of titanium dioxide nanoparticles. J Immunotoxicol 8: 56-67.

31. Du J, Zhang YS, Hobson D, Hydbring P (2017) Nanoparticles for immune system targeting. Drug Discov Today S1359-6446: $30156-3$.

32. Farace C, Sanchez-Moreno P, Orecchioni M, Manetti R, Sgarrella F, et al. (2016) Immune cell impact of three differently coated lipid nanocapsules: pluronic, chitosan and polyethylene glycol. Sci Rep 6: 18423.

33. Lee V, McMahan RS, Hu X, Gao X, Faustman EM, et al. (2015) Amphiphilic polymer-coated CdSe/ZnS quantum dots induce pro-inflammatory cytokine expression in mouse lung epithelial cells and macrophages. Nanotoxicology 9: 336-43.

34. Nakanishi K, Tsukimoto M, Tanuma S, Takeda K, Kojima S (2016) Silica nanoparticles activate purinergic signaling via P2X7 receptor in dendritic cells, leading to production of pro-inflammatory cytokines. Toxicol In Vitro 35: 202-11.

35. Sang X, Zheng L, Sun Q, Li N, Cui Y, et al. (2012) The chronic spleen injury of mice following long-term exposure to titanium dioxide nanoparticles. J Biomed Mater Res A 100: 894-902.

36. Teixeira LK, Fonseca BP, Barboza BA, Viola JP (2005) The role of interferon-g on immune and allergic responses. Memórias do Instituto Oswaldo Cruz 100: 137-44.

37. Yang YW, Luo WH (2016) Cellular biodistribution of polymeric nanoparticles in the immune system. J Control Release 227: 82-93.

38. Chen J, Dong X, Zhao J, Tang G (2009) In vivo acute toxicity of titanium dioxide nanoparticles to mice after intraperitioneal injection. J Appl Toxicol 29: 330-7.

39. Li N, Duan Y, Hong M, Zheng L, Fei M, et al. (2010) Spleen injury and apoptotic pathway in mice caused by titanium dioxide nanoparticules. Toxicol Lett 195 : 161-8.

40. Park EJ, Jeong U, Yoon C, Kim Y (2017) Comparison of distribution and toxicity of different types of zinc-based nanoparticles. Environ Toxicol 32: 1363-74.

41. Iavicoli I, Fontana L, Leso V, Corbi M, Marinaccio A, et al. (2017) Subchronic exposure to palladium nanoparticles affects serum levels of cytokines in female Wistar rats. Hum Exp Toxicol 960327117702952.

42. Liu Y, Jiao F, Qiu Y, Li W, Qu Y, et al. (2009) Immunostimulatory properties and enhanced TNF- alpha mediated cellular immunity for tumor therapy by $\mathrm{C} 60(\mathrm{OH}) 20$ nanoparticles. Nanotechnology 20: 415102. 


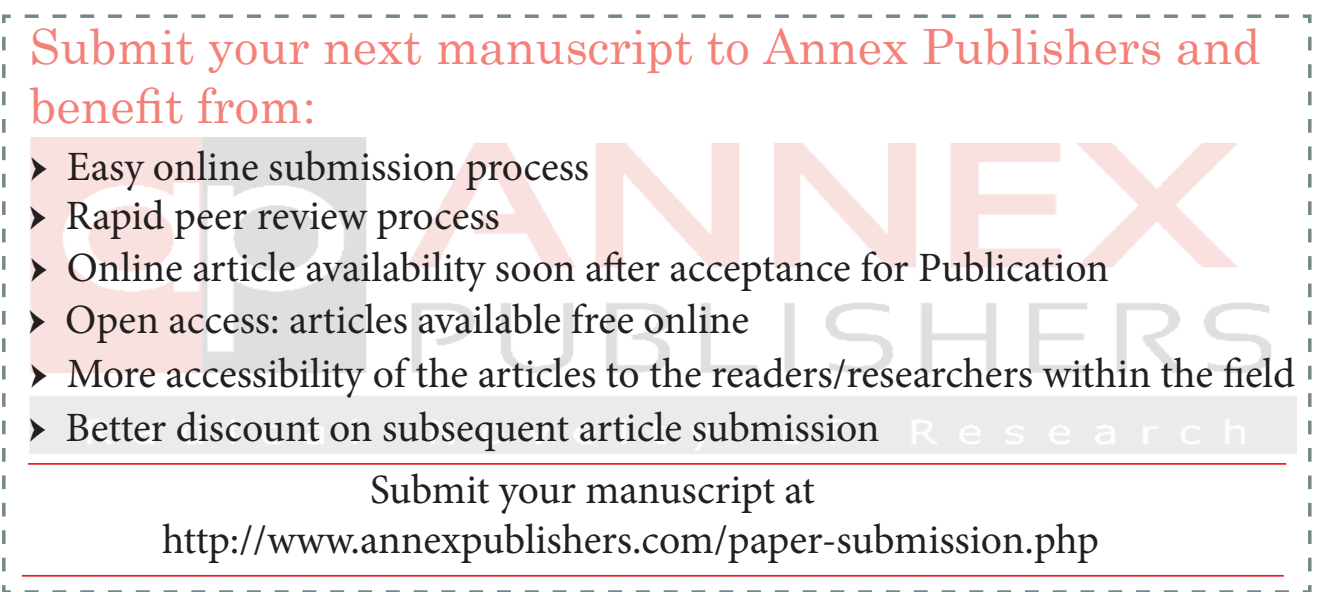

